\title{
DYNAMIC FuZzY LOGIC TO IDENTIFY SAFETY STOCK IN DAIRY INDUSTRIES / EMPIRICAL CASE STUDY
}

\author{
Riyadh Jamegh, Dr. AllaEldin Kassam \& Dr. Sawsan Sabih
}
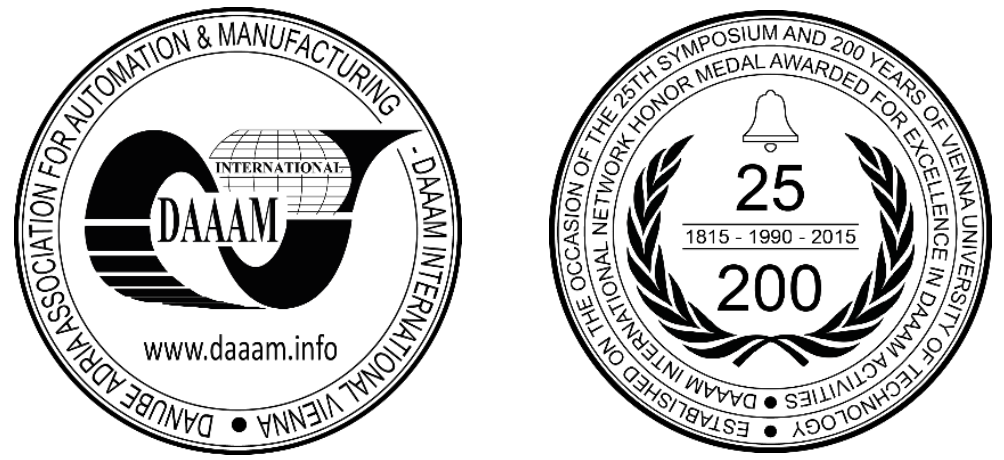

This Publication has to be referred as: Jamegh, R[iyadh]; Kassam, A[llaEldin] \& Sabih, S[awsan] (2018). Dynamic Fuzzy Logic to Identify Safety Stock in Dairy Industries / Empirical Case Study, Proceedings of the 29th DAAAM International Symposium, pp.0983-0992, B. Katalinic (Ed.), Published by DAAAM International, ISBN 978-3-90273420-4, ISSN 1726-9679, Vienna, Austria

DOI: $10.2507 / 29$ th.daaam.proceedings.141

\begin{abstract}
Safety stock (SS) optimization is considered an important element in supply chain inventory optimization especially in small and medium enterprises (SME). In this paper we employee dynamic fuzzy logic process as innovation step to identify safety stock level in dairy industries with objective of minimizing total cost and meet with customer requirements. The proposed model consisting of three main steps firstly, building of fuzzy set for each variable, secondly, identifying max values which represent universe of discourse of variables and identify daily input values of the variables, finally, Execution of proposed model in fuzzy logic tool embedded in Matlab software. The research works to address the problem of increasing storage and increase of holding cost by determining the ideal level of safety stock and this is one of the most important points that the search works. As traditional approaches, which is use statistical models in determining SS level always give poor results about SS level and these is the most important constraints of traditional approaches, therefore, in this paper we have employed advanced methods in solving this problem by employing one of the tools of soft computing (fuzzy logic) to control the levels of safety stock. The researcher tries to build robust and powerful fuzzy model which employee expert knowledge in problem solution. The recommended solution provided in this search demonstrates the validity of proposed model by noting the reduction in the level of safety stocks in real environment.
\end{abstract}

Keywords: dynamic inventory optimization; dynamic fuzzy logic; safety stock optimization; dairy industries

\section{Introduction}

Traditionally, the models and approaches of supply chain management are useful when there is small data to be processed and the complexity of the problem is low. But the challenge emerges with more date system [1]. The industrial enterprises try always to meet with customer needs by improving their service level, so high safety stock level are required, on the other hand, the increasing of safety stock level leads to surplus capital commitment which influences on the economic efficiency. The competing logistic objectives i.e. high capability to delivery and low inventory level describe what called "dilemma of inventory management" [2]. 
Generally, safety stock level is used in supply chain to compensate shortage quantities when the actual demand is more than expected demand, during lead time or when actual lead time longer than expected lead time [3]. Therefore, proper safety stock management as one of the most significant drivers of inventory is considered critical objective in order to have leanness philosophy and toward efficient inventory control [4]. Moreover, proper management of inventory requires suitable safety stock so as to protect against the increasing of stretch in breaking points of the supply chain, which is lead to possible reduction of inventory [5]. The firms under supply chains opinions are working under pressure to provide high customer service level while operating effectively with low inventory level, at the same time the supply chains generally are exposed to different risks types, such as demand uncertain, supply uncertain, process uncertain, lead times uncertain, and disasters risks. Different strategies have been employed to mitigate these risks impact, such as safety stocks, safety time or a combination of these two types [6]. Through this area of inconsistency and conflict, different approaches were proposed in order to determine the adequate inventory. Most of these approaches calculate safety-stock level based on common statistical equations (e.g. demand standard deviation, mean demand rate), so it is just forecasting on the historical data [2]. This, of course, results in a marked weakness in results and also overlooks the dynamic behaviour of current global and competitive markets. A few researches were used advanced approach to identify safety stock so as to overcome the problem of the increase in levels of safety stock due to use of traditional methods. The new approach rely on soft computing to provide methodologies that rely mostly on the fuzzy logic to calculate safety stock and thus provide a dynamic behaviour that take into account the historical data as well as changes that get to key variables such as the level of demand and service level of customer. The aim of this research is to provide dynamic approach to identify safety stock and it can be implanted in different industries.

\section{Literature review}

In (2005) Simchi-Levi and Zhao presented study about effect of safety stock in supply chain by taking three different types of supply chain; first, serial system, second, assembly system, and finally distribution supply chain system. Stochastic service approach (SSA) has been used and continuous review based stock policy employed for each stage. The Poisson distribution of demand was followed and lead time is exogenously determined [7]. Vanteddu et al. (2007) studied the placement of safety stock in supply chain taking into account total cost and response time from the design stage. This model enables the manager of aids supply chain in attain superior strategic fit of individual business strategies and overall supply chain strategies in terms of high cost efficiency and better responsiveness [8].

For safety sock optimization, Jung et al. (2008) used discrete event simulation and linear programming to evaluate and control safety stock and service level along the stages. The linear programming model uses safety stock and service level as decision variables. Determination of safety stock of multiple stage supply chain was done by linear programming model. The proposed model addresses safety stock capacity that must be provided within production capacity in order to have certain appropriate level of safety stock at each production sites [9]. Batel (2010) used optimization model to identify safety stock level in production and distribution zones of supply chain, the researcher identify six variables to be input variable to simulation system, these variables are; work in progress, production start rate, distribution inventory, production rate, waste rate, and distribution rate. The simulation model was executed for 60 runs, the affects six variables of system were obtained and the researcher concluded that impact of available inventory on pre-defined six variables. During implementation of simulation process for aluminium industry, it is noted that the manufacture must capture 120000 units as safety stock for 30 month. The results ensure that the increasing of stock will reduce the load on production variables. also he was suggested that most important for this supply chain to maintain optimal level of safety stock at distribution points [10].

Employing of fuzzy logic theory to identify supply chain safety stock was used by H. R. Rezaei (2012). A weighted association rule is used to determine the weights of variables instead of the equally importance (i.e. with the same weight) to obtain higher accuracy results. There are three input variables which are Weighted- Fast Slow-Non-moving, weighted cost, and lead time, all of these variables used Gaussian membership function while one output i.e. safety stock with triangular membership function. The integration of weighted approach and fuzzy logic was provided to improve intelligent approach to control inventory. It is worth mentioning that the researcher stressed the need to achieve optimization of membership functions by using different algorithms such as genetic algorithm or neural networks [11]. Placement of safety stock in multiple stages supply chain was presented by Youssef Boulaksil (2016). Demand uncertainty and backorder of unmet demand are the two assumption of model. The simulation technique has been employed and the mathematical models solved by rolling horizon. Implementation of proposed model in real number case study, the researcher concluded that the large part of the safety stock should be in downstream to meet the requirements of the customers, and concluded results shows that the safety stock can only be moved to upstream zone when the lead time is relatively low, and also when less uncertainty in customer demand [6].

The relationship between demand uncertainty and inventory level in fast moving consumer goods was studied by Ndivhuwo Nemtajela \& Charles Mbohwa (2017). A survey questionnaire used and distributed to five company and just 255 respondent questionnaire were analysed, the results shows that about $72.2 \%$ of answers is to agree with the statement (there are a strong positive impact between demand uncertainty and suitable inventory management, hence higher uncertainty demand considered more difficult and great challenging of holding inventory in an organization). They also concluded that the organizations should implement effective forecasting techniques [12]. According to our survey about safety stock, it is clear to notice the scarcity of research that deals with safety stock by using fuzzy logic. 
Table (1) below shows the approaches used in different researches to calculate safety stock level;

\begin{tabular}{|l|l|l|l|l|l|}
\hline No. & Researcher & Year & References & $\begin{array}{l}\text { Statistical } \\
\text { approach }\end{array}$ & $\begin{array}{l}\text { Fuzzy } \\
\text { approach }\end{array}$ \\
\hline 1 & $\begin{array}{l}\text { Simchi-Levi\& } \\
\text { Zhao }\end{array}$ & 2005 & {$[7]$} & $<$ & \\
\hline 2 & Vanteddu. & 2007 & {$[8]$} & $<$ & \\
\hline 3 & Jung & 2008 & {$[9]$} & $<$ & \\
\hline 4 & Batel & 2010 & {$[10]$} & $<$ & \\
\hline 5 & H. R. Rezaei & 2012 & {$[1]$} & & $<$ \\
\hline 6 & $\begin{array}{l}\text { Youssef } \\
\text { Boulaksil }\end{array}$ & 2016 & {$[6]$} & $<$ & \\
\hline 7 & $\begin{array}{l}\text { Ndivhuwo } \\
\text { Nemtajela }\end{array}$ & 2017 & {$[12]$} & $<$ & \\
\hline
\end{tabular}

Table 1. Approaches used to calculate safety stock level

\section{Fuzzy logic process}

The first emerge of fuzzy logic was return to Zadeh in (1965) [13]. Fuzzy logic is a nonlinear mathematical model used to deal with uncertainty and ambiguity condition to map input data into crisp output. Fuzzy logic system incorporate human knowledge with computer software in order to it an engineering manner to control ill-defined systems by nonlinearity modelling approach [14]. Fuzzy logic system consists of four components [15]; as shown in figure (1);

First. Knowledge base: - It is an important part of the fuzzy logic system, knowledge base consist of database i.e. (linguistic variables, membership function and rules) knowledge base consisting of three components, these are linguistic variable, membership function, and rules

i) Linguistic variables: - Description of variables in words instead of value called linguistic variables, this variables will assigning to the variables of system (input \& output) and it is decomposed into terms represent the cases of the variable.

ii) Membership function (MF):- Membership function is the second step to create Knowledge base, which represent specific shape used to define fuzzy set features by assigning for the corresponding values the of elements i.e. degree of membership function, their major function is to map input crisp values to membership interval [0-1]

iii) The rules:-The rules used to identify output values, it consist of antecedent and consequent which is written by If then manner. Rules can drive by two methods, first extraction of rules from expert domain, second extraction of rules from data.in our approach, rules based experts knowledge were extracted.

Second: Fuzzification process:- a Fuzzification is the process of transfer crisp value to equivalent membership values of the suitable fuzzy set via membership function, through this process the output represent degree of membership function for related fuzzy linguistic with the interval ranging of [0-1].

Thirdly: Inference engine:- The inference engine is responsible of mapping from fuzzy input to the fuzzy output by using IF-THEN type fuzzy rules. The rules will reflect a mechanism of human reasoning.

Fourthly: Defuzzification: is defined as process of reducing an aggregated (or clipped) fuzzy set to a crisp number, this value presumably to be the most representative value of that fuzzy set interval

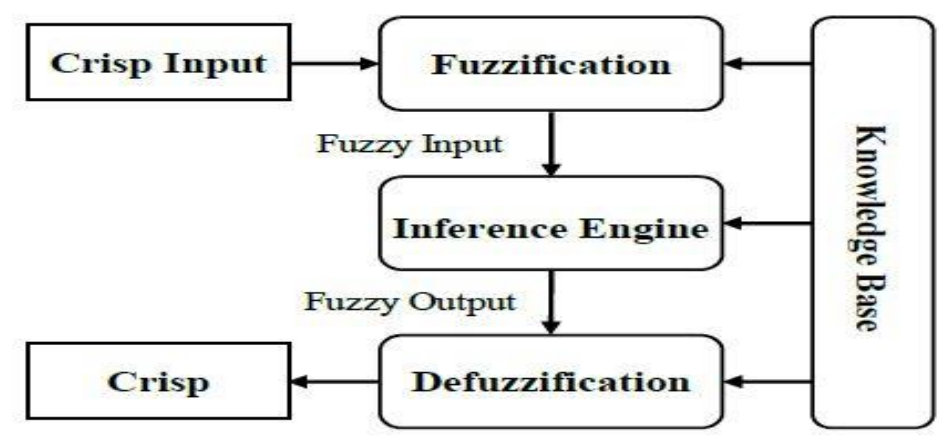

Fig. 1. Components of fuzzy logic system [15] 


\section{Proposed approach}

Now, the architecture of proposed approach is presented. The proposed approach consist of three steps: Identifying of demand uncertainty conditions, identifying of raw material conditions, and identifying of inventory on hand conditions in order to develop dynamic fuzzy logic safety stock, as shown in figure (2);

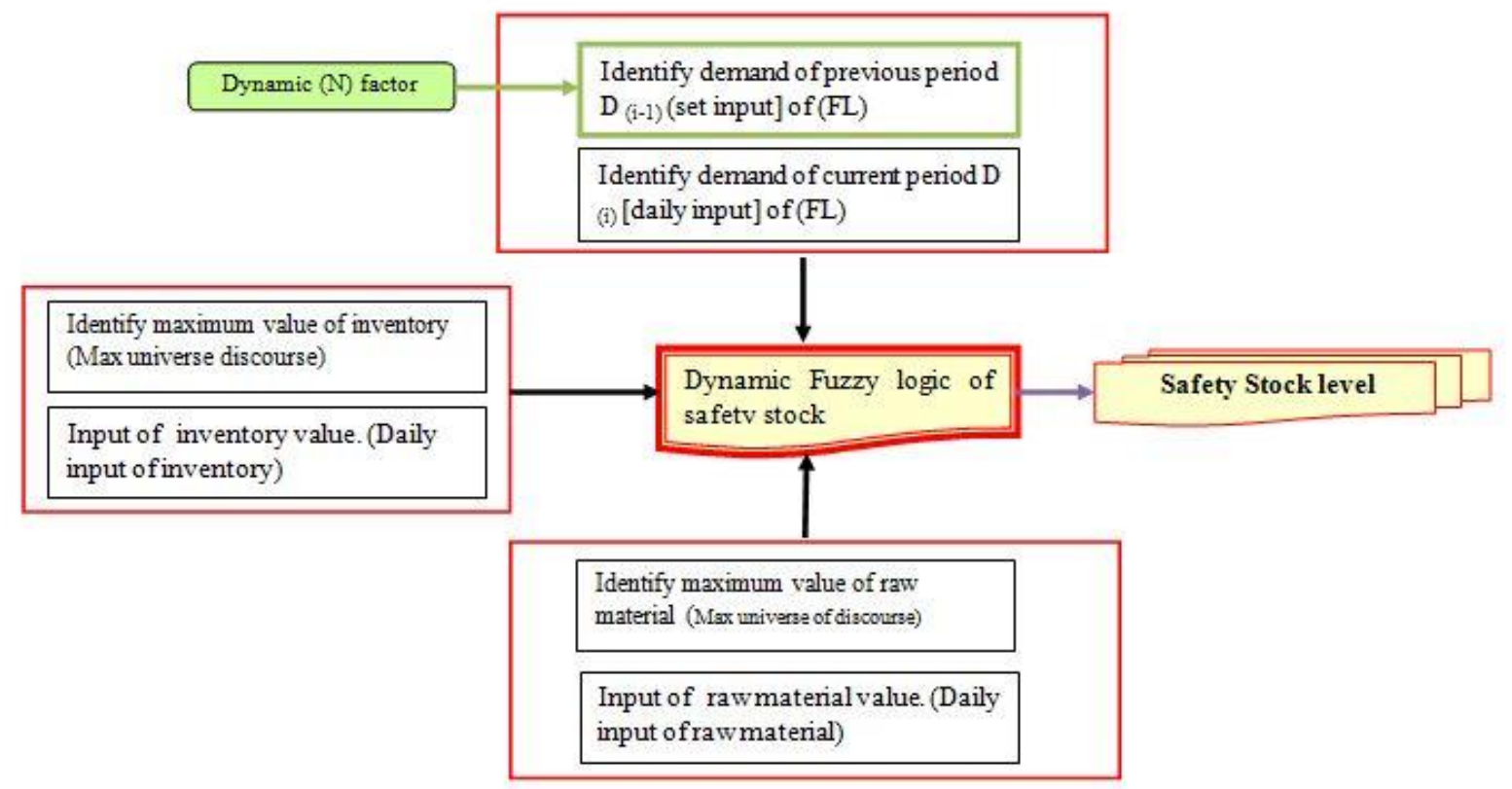

Fig. 2. General architecture of proposed model

\subsection{Demand uncertainty conditions}

Demand uncertainty play vital role in identifying safety stock. In order to cope with dynamic and competitive of global market, dynamic fuzzy safety stock model is suggested. The uncertainty of demand is embedded in this approach by following these steps;

- Identifying of dynamic factor ( $\mathrm{N}$ factor) which represent max rate of increasing in demand for specific period (monthly period are identified).

- Building of membership function set to describe the statues of demand as shown in figure 3 which represents fuzzy sets of demand stability level

- Identify max value of universe discourse for first variable (demand stability level) of current day (D(i)) by multiplying $(\mathrm{N})$ factor by demand of previous day $(\mathrm{D}(\mathrm{i}-1))$.

- Input of value of demand of current day (D(i) ).

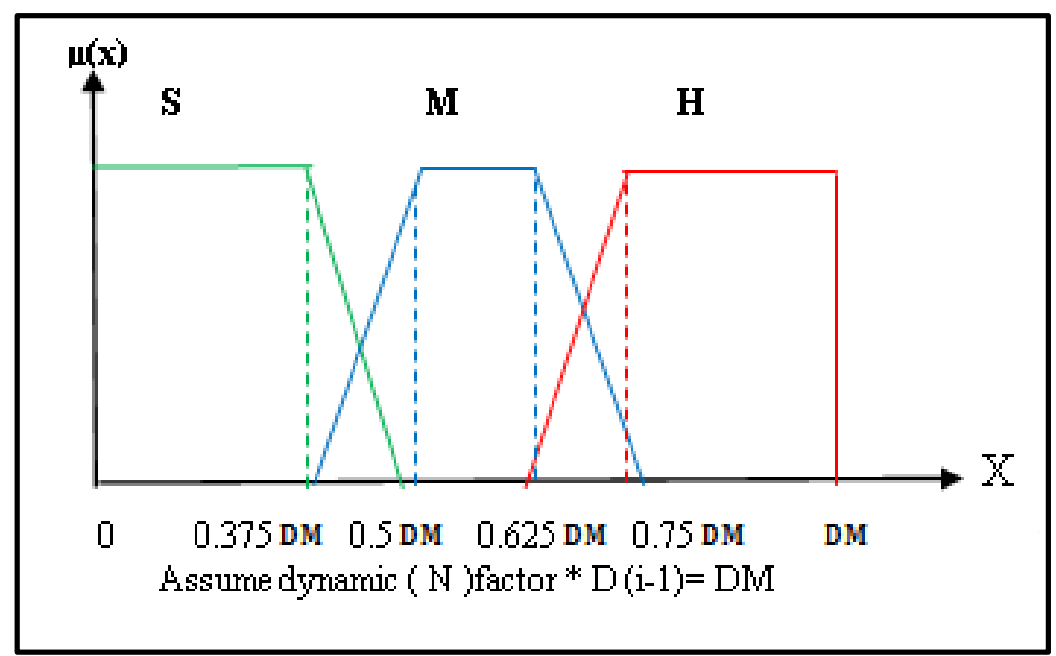

Fig. 3. Fuzzy sets of demand stability level (first variable) 
The reason behind choose trapezoidal shape return to exist of period in which the demand can be described as a stable which makes us benefit from the stability advantage provided by this type of function due to the confidence area in the middle of the shape.

\subsection{Raw material conditions}

Raw material availability is necessary in identifying safety stock level because unavailability of raw material leads to unmet customer requirement and reflect bad reputation of company. The raw material is embedded by following steps below;

- Building of set of membership function to describe the statues of raw material availability. These sets are unavailability (UAV), rare availability (RAV), and availability (AV) as shown in figure 4 .

- Identifying of max value and min values of raw material availability to represents universe discourse of the variable for specific month.

- Input of daily value of raw material availability of current day $\mathrm{D}(\mathrm{i})$.

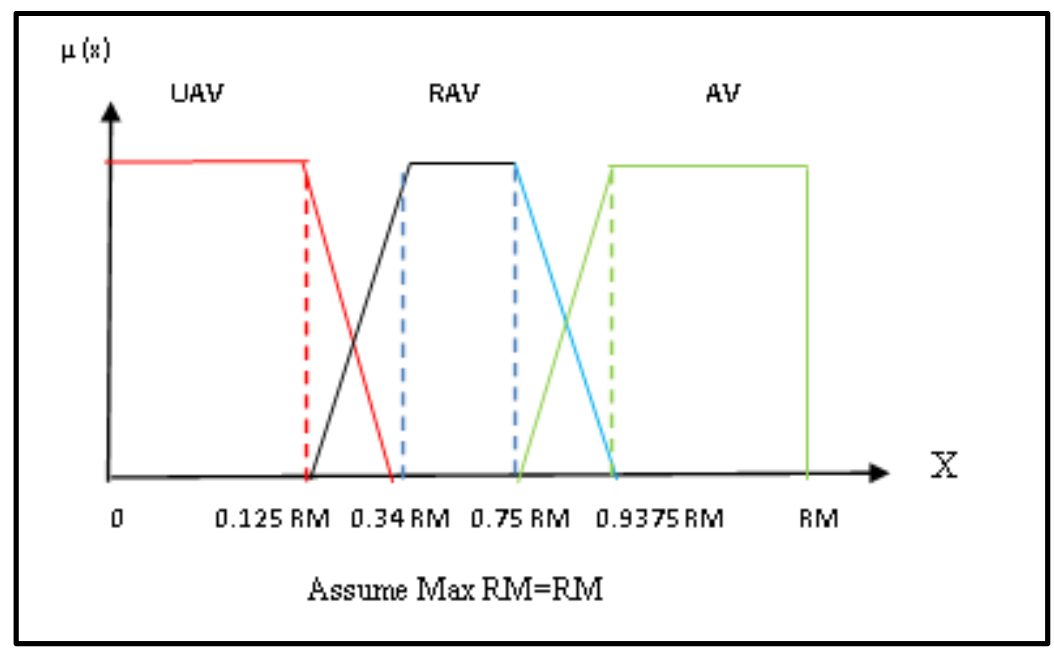

Fig. 4. Fuzzy sets of raw material availability (second variable)

\subsection{Inventory on hand conditions}

The quantity of on hand inventory product must be taken in account to identify safety stock level where a suitable inventory management is lead to reduction in safety stock level. The procedure of identifying on hand inventory is embedded in this approach by following steps below;

- Building of set of membership function to describe the statues of on hand inventory which are low, medium, and high as shown in figure (5).

- Identifying of max value and min values of on hand inventory to represents universe discourse of the variable for specific month.

- Input of daily value of on hand inventory of current day (D(i).

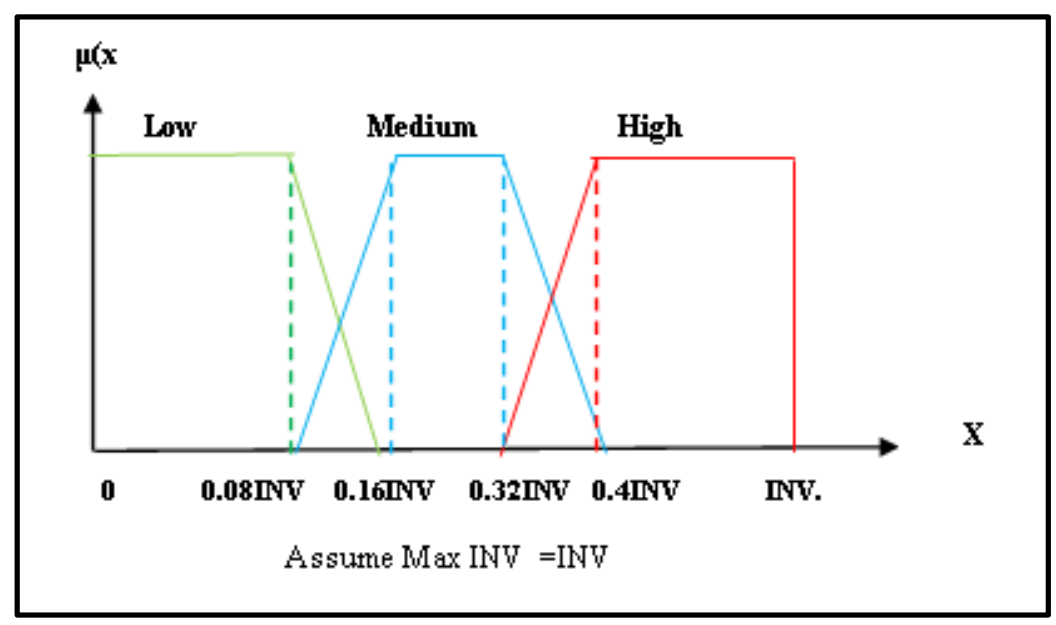

Fig. 5. Fuzzy sets of inventory on hand (third variable) 


\subsection{Safety stock level}

Safety stock level is the only output of this system. The reasoning rules are applied to the system after the entering of all values of input variables. The concept of period specification play vital role in identifying safety stock level. Period specification represents the season conditions of the selected product and it has been classified into three types which are peak, normal, and weak period. Max value of universe discourse of safety stock level are changed daily which allowing high flexibility for system and dynamic statues based on period specification and this change of max value will done by executing the following steps.

- Identifying of percentage value of safety stock which ranging from (10-30)\% of current period demand $\mathrm{D}(\mathrm{i})$, and the value chosen based on period specification, the value is (10\%) for weak period, (20)\% for normal period, and (30)\% for peak period

- Building of set of membership function to describe the statues of safety stock level as shown in figure 6 fuzzy sets of safety stock

- Identifying of max value of universe discourse as a percentage of demand of current day (D(i) ) for specific period specification and this value is changed daily.

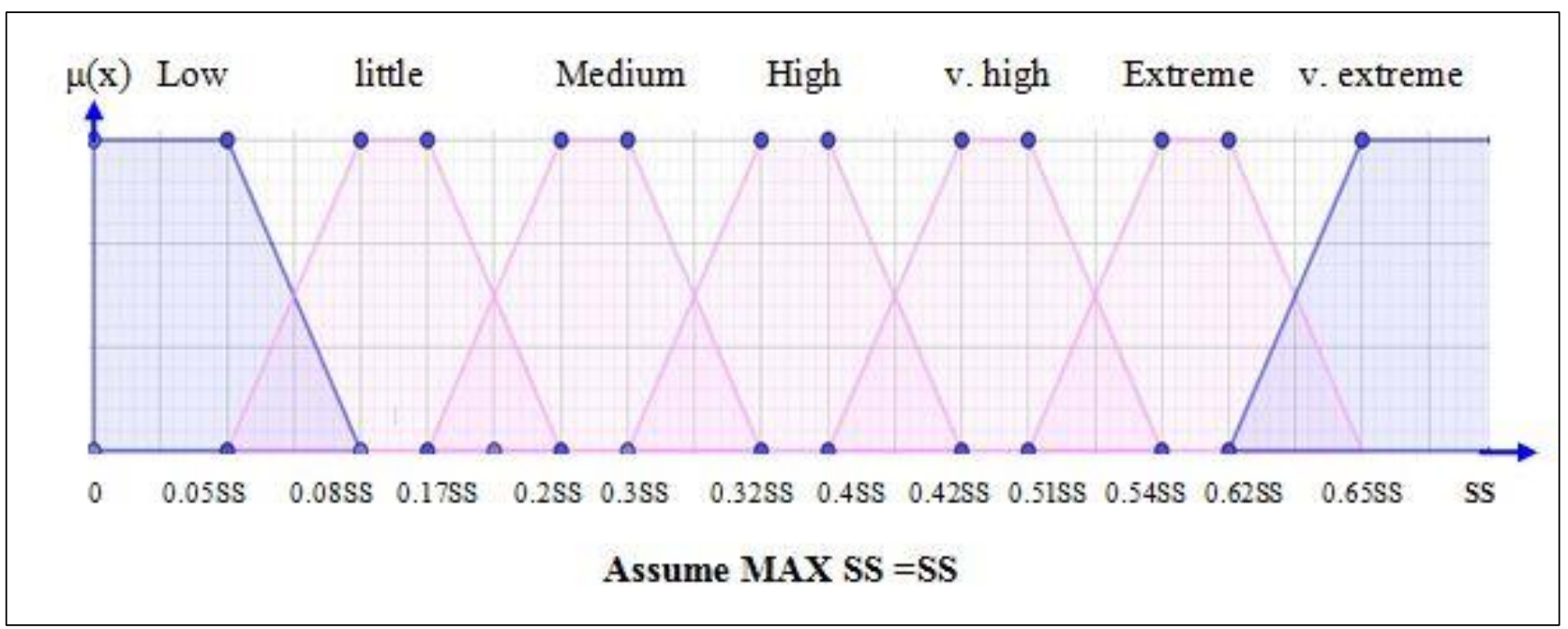

Fig. 6. Fuzzy sets safety stock (unique output)

\section{Case Study}

The validity of proposed model is demonstrated by applying numerical example. Dairy industries considered appropriate field to test the model. The reasons behind that is specifications related to the dairy industry which are dynamic environment, short shelf life of product, and high competitive environment, two products were selected, soft cheese $400 \mathrm{gm}$ and yogurt $400 \mathrm{gm}$. The steps below show the implementation of model.

\subsection{Step (1); Demand Uncertainty Conditions (Input Variable 1)}

In this step, universe discourse of the variable is identified by multiplying ( $\mathrm{N}$ factor) by previous demand $\mathrm{D}(\mathrm{i}-1)$. ( $\mathrm{N}$ factor) must identified by comparing the demand of first day with demand of second day, then second day with third and so on, then select max percentage increasing value to represents ( $\mathrm{N}$ factor). Table (2) shows the universe discourse of this variable. These values are changed daily automatically in order to identify SS level of two selected products.

\begin{tabular}{|c|c|c|c|}
\hline System variables & $\begin{array}{c}\text { Linguistic } \\
\text { variable }\end{array}$ & Linguistic values & Numerical ranges \\
\hline \multirow{2}{*}{$\begin{array}{c}\text { Demand } \\
\text { uncertainty } \\
\text { condition }\end{array}$} & Low & $\mathrm{L}$ & $(0-0.5 \mathrm{DM})$ \\
\cline { 2 - 4 } & Medium & $\mathrm{M}$ & $(0.375-0.75 \mathrm{DM})$ \\
\cline { 2 - 4 } & High & $\mathrm{H}$ & $(0.625-1 \mathrm{DM})$ \\
\hline
\end{tabular}

Table 2. Values of fuzzy set for demand stability level

For example, the max value of universe discourse for third day equal to $(2 * 65=130)$ where $(2)$ represents $(\mathrm{N}$ factor) for soft cheese in January month and (65) represents the demand of second day, table (3) shows the results of demand uncertainty universe discourse. 


\begin{tabular}{|c|c|c|c|}
\hline System variables & $\begin{array}{l}\text { Linguistic } \\
\text { variable }\end{array}$ & Linguistic values & Numerical ranges \\
\hline \multirow{3}{*}{ Demand uncertainty condition } & Low & $\mathrm{L}$ & $(0-0.5) * 130$ \\
\hline & Medium & $\mathrm{M}$ & $(0.375-0.75) * 130$ \\
\hline & High & $\mathrm{H}$ & $(0.625-1) * 130$ \\
\hline
\end{tabular}

Table 3. Applied values for first variable fuzzy set

\section{2 step (2); Raw Material Availability Conditions (Input Variable 2)}

Universe discourse of raw material are identified monthly basis and daily level of raw material is entered to the model in order to identify the impact of this variable on SS level. Table (4) shows the universe discourse of this variable while table (5) presents the value of universe discourse of the variable in January month where max level of raw material is (30ton).

\begin{tabular}{|c|c|c|c|}
\hline System variables & Linguistic variable & Linguistic values & Numerical ranges \\
\hline \multirow{4}{*}{ Raw material availability } & Unavailable & $\mathrm{UAV}$ & $(0-0.34 \mathrm{RM})$ \\
\cline { 2 - 4 } & Rare available & $\mathrm{RAV}$ & $(0.125-0.9375 \mathrm{RM})$ \\
\cline { 2 - 4 } & Available & $\mathrm{AV}$ & $(0.75-1 \mathrm{RM})$ \\
\hline
\end{tabular}

Table 4. Values of fuzzy set for raw material availability

\begin{tabular}{|c|c|c|c|}
\hline System variables & Linguistic variable & Linguistic values & Numerical ranges \\
\hline \multirow{3}{*}{ Raw material availability } & Unavailable & UAV & $(0-0.34) * 30$ \\
\cline { 2 - 4 } & Rare available & RAV & $(0.125-0.9375) * 30$ \\
\cline { 2 - 4 } & Available & AV & $(0.75-1) * 30$ \\
\hline
\end{tabular}

Table 5. Applied values for second variable fuzzy set

\section{3 step (3); Inventory On Hand Conditions (Input Variable 3)}

Inventory on hand universe discourse and fuzzy sets are identified in this step, Table (6) shows the universe discourse of this variable and table (7) presents the value of universe discourse of the variable in January month where max level of raw material is (75 package).

\begin{tabular}{|c|c|c|c|}
\hline System variables & Linguistic variable & Linguistic values & Numerical ranges \\
\hline \multirow{3}{*}{ On hand inventory } & Low & $\mathrm{L}$ & $(0-0.16 \mathrm{INV})$ \\
\cline { 2 - 4 } & Medium & $\mathrm{M}$ & $(0.08-0.4 \mathrm{INV})$ \\
\cline { 2 - 4 } & High & $\mathrm{H}$ & $(0.32-1 \mathrm{INV})$ \\
\hline
\end{tabular}

Table 6. Values of fuzzy set for inventory on hand

\begin{tabular}{|c|c|c|c|}
\hline System variables & Linguistic variable & Linguistic values & Numerical ranges \\
\hline \multirow{3}{*}{ On hand inventory } & Low & $\mathrm{L}$ & $(0-0.16) * 75$ \\
\cline { 2 - 4 } & Medium & $\mathrm{M}$ & $(0.08-0.4) * 75$ \\
\cline { 2 - 4 } & High & $\mathrm{H}$ & $(0.32-1) * 75$ \\
\hline
\end{tabular}

Table 7. Applied values for third variable fuzzy set

\section{4 step (4); Safety Stock Level (Output)}

The only one output i.e. safety stock level is determined by this model, seven sets are used to identify grip the SS level. It is crucial to identify period specification. In January for the soft cheese the period is peak, so the percentage is(30\%) of $\mathrm{D}(\mathrm{i})$, while for yogurt $400 \mathrm{gm}$ the period specification is $(20) \%$ of $\mathrm{D}$ (i). for example, the max universe discourse of third day of January to soft cheese is $(0.3 * 100)$ where $(0.3)$ represents the period specification value and (100) represents the demand of third day. Table (8) shows the universe discourse of SS level while table (9) presents the value of universe discourse of the soft cheese in January month where max level of SS is changed daily. 


\begin{tabular}{|c|c|c|c|}
\hline System variables & Linguistic variable & Linguistic values & Numerical ranges \\
\hline \multirow{4}{*}{ Safety stock level } & Low & L & $(0 .-0.08 \mathrm{SS})$ \\
\cline { 2 - 4 } & Little & LI & $(0.05-0.2 \mathrm{SS})$ \\
\cline { 2 - 4 } & Medium & $\mathrm{M}$ & $(0.17-0.32 \mathrm{SS})$ \\
\cline { 2 - 4 } & High & $\mathrm{H}$ & $(0.3-0.42 \mathrm{SS})$ \\
\cline { 2 - 4 } & Very high & VH & $(0.4-0.54 \mathrm{SS})$ \\
\cline { 2 - 4 } & Extreme & $\mathrm{E}$ & $(0.51-0.65 \mathrm{SS})$ \\
\cline { 2 - 4 } & Very extreme & VE & $(0.62-1 \mathrm{SS})$ \\
\hline
\end{tabular}

Table 8. Values of fuzzy set for safety stock level

\begin{tabular}{|c|c|c|c|}
\hline System variables & Linguistic variable & Linguistic values & Numerical ranges \\
\hline \multirow{4}{*}{} & Low & L & $(0 .-0.08) * 30$ \\
\cline { 2 - 4 } & Little & LI & $(0.05-0.2) * 30$ \\
\cline { 2 - 4 } & Medium & M & $(0.17-0.32) * 30$ \\
\cline { 2 - 4 } & High & H & $(0.3-0.42) * 30$ \\
\cline { 2 - 4 } & Very high & VH & $(0.4-0.54) * 30$ \\
\cline { 2 - 4 } & Extreme & E & $(0.51-0.65) * 30$ \\
\cline { 2 - 4 } & Very extreme & VE & $(0.62-1) * 30$ \\
\hline
\end{tabular}

Table 9. Applied values for safety stock variable fuzzy set

For the first product (soft cheese) at the first month (January), when calculate SS for third day. The demand of previous equal to (65) package with inventory on hand equal to (9) package and raw material available of (30) tone of raw milk, and demand of current day is (100) package, then the safety stock is (22) package. Table (10) summarize these details

\begin{tabular}{|l|l|l|l|l|l|l|l|l|l|}
\hline Product & month & Day(i) & $\begin{array}{l}\text { Factor } \\
\text { (n) }\end{array}$ & d(i) & Max.RM & daily.RM & Max.INV. & $\begin{array}{l}\text { Daily. } \\
\text { INV. }\end{array}$ & $\begin{array}{l}\text { \% of } \\
\text { d(i) }\end{array}$ \\
\hline P1 & $\mathrm{T} 1$ & 2 & 2 & 65 & 30 & 18.334 & 75 & 9 & 0.3 \\
\hline P1 & $\mathrm{T} 1$ & 3 & 2 & 100 & 30 & 28.635 & 75 & 22 & 0.3 \\
\hline
\end{tabular}

Table 10. Sample of data which feeding to the model

The inferred rules are (19 \&20), and because of using (AND) logic operator, the min values are selected for implication process. Implication process for identifying SS level for third day of January for soft cheese, consisting of three steps, first step is to enter the input values; second step is applying of inference rules of input parameters, while the third step is aggregation of outputs of inference rules.

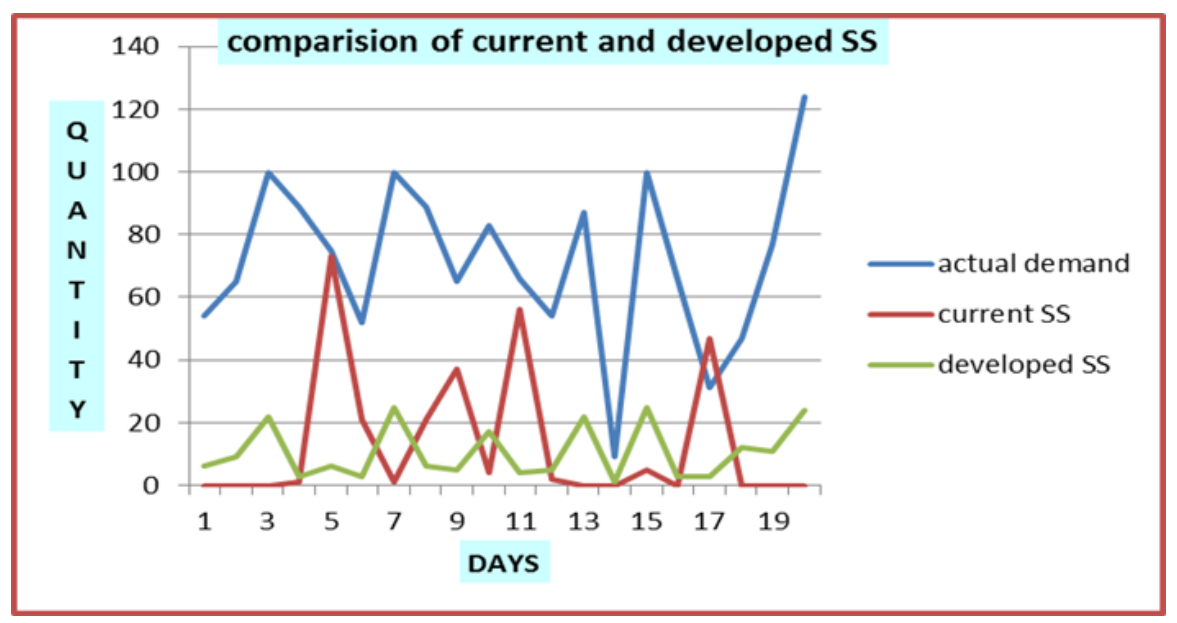

Fig. 7. Safety stock level for proposed and current model 
Table (11) below shows the demand of products and safety stock level for January month for (soft cheese) and (yogurt 4oogm) for company before and after implementation of model. It is easy to notice that the company is putting large quantities of safety stocks to control the varity in demand where the company believes that increasing the level of inventory for day (i) may reduce the pressure on the beginning production rate of the second day (i+1) and put the company in a comfortable position, but this corresponds to the probability of inecreasing demand on the second day which may leads to risk of increasing inventory level, and then increasing of holding cost.

\begin{tabular}{|c|c|c|c|c|c|c|}
\hline Day & $\begin{array}{c}\text { Soft } \\
\text { cheese } \\
\text { demand }\end{array}$ & $\begin{array}{c}\text { Soft } \\
\text { cheese SS } \\
\text { (current) }\end{array}$ & $\begin{array}{c}\text { Soft cheese } \\
\text { SS } \\
\text { (developed) }\end{array}$ & $\begin{array}{l}\text { yogurt } \\
\text { demand }\end{array}$ & $\begin{array}{l}\text { yogurt SS } \\
\text { (current) }\end{array}$ & $\begin{array}{c}\text { yogurt SS } \\
\text { (developed) }\end{array}$ \\
\hline 1 & 54 & 0 & 6 & 146 & 192 & 4 \\
\hline 2 & 65 & 0 & 9 & 70 & 122 & 1 \\
\hline 3 & 100 & 0 & 22 & 246 & 84 & 21 \\
\hline 4 & 89 & 1 & 3 & 85 & 14 & 2 \\
\hline 5 & 75 & 73 & 6 & 301 & 16 & 25 \\
\hline 6 & 52 & 21 & 3 & 195 & 81 & 3 \\
\hline 7 & 100 & 1 & 25 & 63 & 18 & 1 \\
\hline 8 & 89 & 21 & 6 & 257 & 8 & 22 \\
\hline 9 & 65 & 37 & 5 & 235 & 99 & 4 \\
\hline 10 & 83 & 4 & 17 & 198 & 164 & 3 \\
\hline 11 & 66 & 56 & 4 & 180 & 304 & 3 \\
\hline 12 & 54 & 2 & 5 & 278 & 334 & 4 \\
\hline 13 & 87 & 0 & 22 & 300 & 34 & 4 \\
\hline 14 & 9 & 0 & 1 & 235 & 102 & 4 \\
\hline 15 & 100 & 5 & 25 & 225 & 174 & 3 \\
\hline 16 & 66 & 0 & 3 & 162 & 320 & 3 \\
\hline 17 & 31 & 47 & 3 & 170 & 350 & 3 \\
\hline 18 & 47 & 0 & 12 & 198 & 182 & 3 \\
\hline 19 & 77 & 0 & 11 & 215 & 267 & 3 \\
\hline 20 & 124 & 0 & 24 & 210 & 251 & 3 \\
\hline & & Sum $=268$ & Sum $=212$ & & Sum $=3116$ & Sum $=119$ \\
\hline & & \multicolumn{2}{|c|}{ Reduction $=20 \%$} & & \multicolumn{2}{|c|}{ Reduction $=96 \%$} \\
\hline
\end{tabular}

Table 11. Demand and safey stock level (current and developed conditions for two products)

After application of the proposed model, it was completely different through a significant decrease in the level of safety stocks in general and makes the company more stable by reducing the amounts of storage and thus reduces the total cost of production and storage of those quantities. Even when there is a drop in demand, the company is away from of expect to high holding costs. Figure 7 shows the impact of proposed model in identify SS level for soft cheese as compared to current inventory level.

Referring to table (11), the level of safety stock, and by the results of comparison for before and after application of the model, it is easy to notice that great impact of model and the reduction in safety stock level is about $20 \%$ for soft cheese and reaches to $96 \%$ reduction for yogurt $400 \mathrm{gm}$.

\section{Conclusion}

Basis on the obtain results by applying the proposed model, you can get the following conclusions; In this research, safety stock level (SSL) is formulated as dynamic fuzzy logic where new algorithm is presented which contain classical feature of fuzzy logic and advanced feature represented by the dynamism behavior of system. As a compared to the traditional approaches of SSL, the proposed model presents great positive impact and significance contribution because the knowledge of experts has been employed in this model in dynamic manner which leads to high accurate results and overcome the problem of poor inventory system where the common statistical equations that are used for calculating safety stocks showed a clear deficit when working in a dynamic environment such as dairy industries.

In industrial field, the organization works to overcome the problem of demand and supply uncertainty by increasing inventory level but this will leads to increase holding cost, while the reduction in inventory level will lead to increases the likelihood of shortage occurrence in fulfillment customer demand, hence the use of advanced scientific approach to determine the optimal level of safety stock considered crucial step for all companies especially for dairy and food industries. 
By using the dynamic fuzzy logic the level of inventory was reduced with range (20-88) \% for different months of year of the two products (soft cheese and yogurt) with achievement of all customer demand. There are some research areas that can be developed in this model, first it can be use neural network to optimize inference rules, and consequently this leads to more accurate results. Also it is very important to check the validity of proposed model in service field.

\section{References}

[1] Y. Madhwal and P. B. Panfilov,(2017) "Blockchain And Supply Chain Management: Aircrafts'parts'business Case," 28th DAAAM International Symposium \& Proceedings, Vienna, Austria, ISSN 1726 9679, ISBN 978902734-11-2, pp.1051-1056, Published by DAAAM International Vienna , DOI 0.2507/28th.daaam.proceedings. 1462017 .

[2] J. Becker, W. Hartmann, S. Bertsch, J. Nywlt, and M. Schmidt,(2013) "Dynamic Safety-Stock Calculation," World Academy of Science, Engineering and Technology, International Science Index 82, International Journal of Mechanical, Industrial Science and Engineering, vol. 7, pp. 781-785.

[3] Z. Yonggang,(2009) "Analyzing on impact factors of safety stock under random requirement," proceding of International Conference on Networks Security, Wireless Communications and Trusted Computing, China, ISBN 978-0-7695-3610-1, pp. 744-747, Publisher: IEEE, Wuhan, Hubei, 10.1109/NSWCTC.2009.189.

[4] B. Amirjabbari and N. Bhuiyan, (2014)"Determining supply chain safety stock level and location," Journal of Industrial Engineering and Management, vol. 7, NO .1, pp. 42-71, Online ISSN: 2014-0953 - Print ISSN: 20148423 DOI: http://dx.doi.org/10.3926/jiem.543 .

[5] B. Amirjabbari,(2011) "An application of a cost minimization model in determining safety stock level and location," World Academy of Science, Engineering and Technology ,79 , pp. 797-806,Concordia University.

[6] Y. Boulaksil,(2016) "Safety stock placement in supply chains with demand forecast updates," Operations Research Perspectives, vol. 3, pp. 27-31, ISSN, 2214-7160, DOI: http://dx.doi.org/10.1016/j.orp.2016.07.001.

[7] D. Simchi-Levi and Y. Zhao,(2005) "Safety stock positioning in supply chains with stochastic lead times," Manufacturing\&Service Operations Management, vol. 7, 2005, pp. 295-318, ISSN, 1523-4614 DOI: http://dx.doi.org/10.1287/msom.1050.0087.

[8] G. Vanteddu, R. B. Chinnam, K. Yang, and O. Gushikin,(2007) "Supply chain focus dependent safety stock placement," International Journal of Flexible Manufacturing Systems, vol. 19, pp. 463-485, ISSN, 19:463-485, DOI: $10.1007 / \mathrm{s} 10696-008-9050-\mathrm{z}$.

[9] J. Y. Jung, G. Blau, J. F. Pekny, G. V. Reklaitis, and D. Eversdyk,(2008) "Integrated safety stock management for multi-stage supply chains under production capacity constraints," Computers \& Chemical Engineering, vol. 32, pp. 2570-2581,ISSN, 2570-2581, DOI: https://doi.org/10.1016/j.compchemeng.2008.04.003.

[10] R. Patel, L. L. Rodrigues, and V. Kamath, (2010)"Optimizing safety stock in manufacturing supply chain management: A system dynamics approach," proceeding for 12th International Conference on Computer Modelling and Simulation, 2010, pp. 386-391, puplisher IEEE, DOI: 10.1109/UKSIM.2010.78.

[11] H. R. Rezaei,(2012) "Developing an Intelligent Inventory Control Model, Applying Fuzzy Logic and Association Rule Mining,", International Journal of Emerging Technology and Advanced Engineering, Volume 2, No. 9, 2012, pp 149-153, ISSN 2250-2459.

[12] N. Nemtajela and C. Mbohwa,(2017) "Relationship between inventory management and uncertain demand for fast moving consumer goods organisations," Procedia 14th Global Conference on Sustainable Manufacturing, South Africa,ISSN, 2351-9789, vol. 8, pp. 699-706, Publisher, Elsevier B.V, Stellenbosch, DOI : 10.1016/j.promfg.2017.02.090.

[13] "L. Zadeh,(1965)" F. Sets," Information and control.-NY, pp. 338-353.

[14] P. Humphreys, A. McCloskey, R. McIvor, L. Maguire, and C. Glackin,(2006) "Employing dynamic fuzzy membership functions to assess environmental performance in the supplier selection process," International Journal of Production Research, vol. 44, No. 12, 2006, pp. 2379-2419, ISSN, 0020-7543 , DOI: 10.1080/00207540500357476.

[15] J. M. Mendel, (1995)"Fuzzy logic systems for engineering: a tutorial," Proceedings of the IEEE, ISSN, 0018-9219/ vol. 83, N.3, pp. 345-377, Publisher: IEEE, DOI: 10.1109/5.364485 . 